\title{
A!
}

This is an electronic reprint of the original article.

This reprint may differ from the original in pagination and typographic detail.

Rinaldi, R.; Guigno, P.; Cingolani, R.; Lipsanen, Harri; Sopanen, M.; Tulkki, J.; Ahopelto, J.

\section{Zeeman effect in parabolic quantum dots}

Published in:

Physical Review Letters

DOI:

10.1103/PhysRevLett.77.342

Published: 08/07/1996

Document Version

Publisher's PDF, also known as Version of record

Please cite the original version:

Rinaldi, R., Guigno, P., Cingolani, R., Lipsanen, H., Sopanen, M., Tulkki, J., \& Ahopelto, J. (1996). Zeeman effect in parabolic quantum dots. Physical Review Letters, 77(2), 342-345.

https://doi.org/10.1103/PhysRevLett.77.342

This material is protected by copyright and other intellectual property rights, and duplication or sale of all or part of any of the repository collections is not permitted, except that material may be duplicated by you for your research use or educational purposes in electronic or print form. You must obtain permission for any other use. Electronic or print copies may not be offered, whether for sale or otherwise to anyone who is not an authorised user. 


\title{
Zeeman Effect in Parabolic Quantum Dots
}

\author{
R. Rinaldi, P. V. Giugno, and R. Cingolani \\ Unità INFM-Dipartimento Scienza dei Materiali, Università di Lecce, Lecce, Italy \\ H. Lipsanen, M. Sopanen, and J. Tulkki \\ Optoelectronic Laboratory, Helsinki University of Technology, 02150 Espoo, Finland \\ J. Ahopelto \\ VTT Electronics, Otakaari 7B, 02150 Espoo, Finland
}

(Received 2 January 1996)

\begin{abstract}
An unprecedentedly well resolved Zeeman effect has been observed when confined carriers moving along a closed mesoscopic path experience an external magnetic field orthogonal to the orbit plane. Large Zeeman splitting of excited higher angular momentum states is observed in the magnetoluminescence spectrum of quantum dots induced by self-organized InP islands on InGaAs/GaAs. The measured effect is quantitatively reproduced by calculations including the vertical quantum well confinement and strain induced, nearly parabolic, lateral confinement, together with the magnetic interaction. [S0031-9007(96)00469-3]

PACS numbers: 71.70.Ej, 73.20.Dx, 78.55.Cr
\end{abstract}

In the limit of strong confinement, the band structure of a semiconductor quantum dot (QD) forms a sequence of deltalike states corresponding to the quantized levels of a "macroatom." The study of the electronic and physical properties of these dots is of great relevance for all branches of fundamental physics. The interaction of an external magnetic field with the quantized levels of the dot has been investigated in field-effect quantum dots [15] and in dots obtained by electron-beam lithography and shallow etching [6]. In this Letter we report evidence of the Zeeman effect occurring in the atomiclike states of parabolic InGaAs/GaAs quantum dots. Magnetoluminescence experiments clearly show the splitting of the interband transitions corresponding to quantum dot states with $n+|m| \leq 5$, where $n$ is the principal quantum number and $m$ is the angular momentum quantum number. The splitting occurs when the magnetic field is parallel to the symmetry axis of the structure. This is attributed to the breaking of the degeneracy of states with different values of the angular momentum quantum number induced by the external magnetic field (Zeeman splitting) [7-9].

State-of-the-art quantum dot samples were fabricated by self-organized growth of InP islands on the topmost barrier of an $\mathrm{In}_{0.1} \mathrm{Ga}_{0.9} \mathrm{As} / \mathrm{GaAs}$ quantum well (QW) [10]. The propagation of the strain field induced by the InP islands through the GaAs barrier layer results in a parabolic potential in the QW plane (see inset in Fig. 1). The samples consisted of a $6.5 \mathrm{~nm} \mathrm{In}_{0.1} \mathrm{Ga}_{0.9}$ As QW and a $5 \mathrm{~nm}$ GaAs cap layer, covered by $\mathrm{InP}$ islands of $20 \mathrm{~nm}$ height and $80 \mathrm{~nm}$ diameter. The resulting depth of the lateral confining potential was about $70 \mathrm{meV}$ for electrons and $25 \mathrm{meV}$ for heavy holes [11].

In Fig. 1 we show the cw photoluminescence spectra as a function of the excitation power density. The spectra show up to five intersubband transitions involving quantized electron and hole states in the dots. At the lowest excitation intensity $(514 \mathrm{~nm}$ line of an argon laser, $I_{\text {exc }}=5 \mathrm{~W} \mathrm{~cm}^{-2}$ ) the spectrum exhibits three well resolved bands due to radiative transitions involving the first three quantized states. The luminescence line at $1.439 \mathrm{eV}$ is the lowest excitonic line of the InGaAs quantum well. This signal arises from the sample area between adjacent

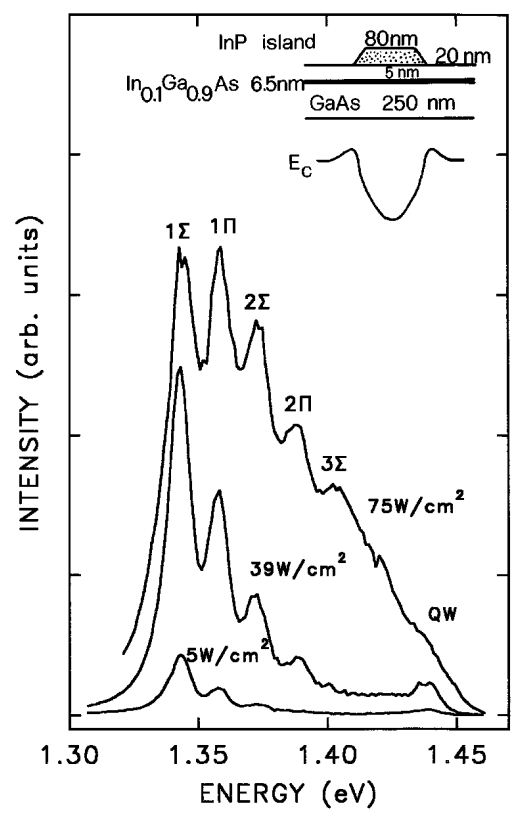

FIG. 1. Photoluminescence spectra of InGaAs quantum dots excited with an argon laser. For $I_{\mathrm{exc}}=75 \mathrm{~W} / \mathrm{cm}^{2}$ we estimate, on the basis of peak intensities, that there are approximately 6-8 electrons and 6-8 holes in a quantum dot. Inset: schematic diagram of the strain-induced confining potential of the dots. 
islands. With increasing excitation intensity, we observe the filling of higher-energy quantized states, up to the fifth level. Under all pumping conditions, the separation of the recombination lines is rather constant (about $17 \mathrm{meV}$ ). The energy position of the observed transitions is consistent with the calculated confinement energies, according to Ref. [12]. Clearly, these dots evidence strong threedimensional confinement properties.

In Fig. 2(a) we report the $\mathrm{cw}$ magnetoluminescence spectra obtained under $I_{\text {exc }}=39 \mathrm{~W} \mathrm{~cm}^{-2}$ excitation intensity and with the magnetic field parallel to the growth axis of the structure $(z)$. The zero field spectrum exhibits four well resolved lines due to intersubband recombination of carriers from the four lowest QD energy levels. With increasing magnetic field the recombination lines corresponding to excited states broaden and split into two or more lines of different amplitude and width. This is clearly observable in Fig. 2(a), where the split transitions arising from the single lines at $0 \mathrm{~T}$ are connected by dashed lines. Note that changing the magnetic field orientation by $90^{\circ}$ ( $B$ parallel to the quantum well layer), the excited transitions in the spectra exhibit neither splitting nor appreciable shift with increasing field, as shown in Fig. 2(b).

In Figs. 3(a) and 3(b) we present the fan plots obtained from the magnetoluminescence spectra under different band filling conditions (i.e., different excitation intensity). All lines corresponding to excited states exhibit clear splitting. The data can only be explained starting from the quantum mechanical treatment of magnetic interaction affecting a particle in a potential of axial symmetry [13], i.e., a system which can be described in terms of cylindrical coordinates.

As shown by us recently [12], the confinement energies of our quantum dot system can be reproduced by the Luttinger-Kohn model. The effective potential for electrons and heavy holes includes the QW confinement potential (vertical confinement) and the strain induced deformation potential (lateral confinement). Because of the axial symmetry, the envelope wave functions can be written as $R_{n m}(r, z) e^{i m \phi}$, where the principal quantum number $n$ specifies the number of radial modes and $m$ the $z$ component of angular momentum $L_{z}=m \hbar$. The states are labeled $n \Sigma, n \Pi^{ \pm}, n \Delta^{ \pm}$for $m=0, \pm 1, \pm 2, \pm 3, \ldots$, where states with the same $|m|$ are degenerate at zero field. Therefore our ground state is $1 \Sigma$, the first excited state $1 \Pi^{ \pm}$, and the second excited state $2 \Sigma$ and $1 \Delta^{ \pm}$(nearly degenerate). Because of the decoupling of heavy and light
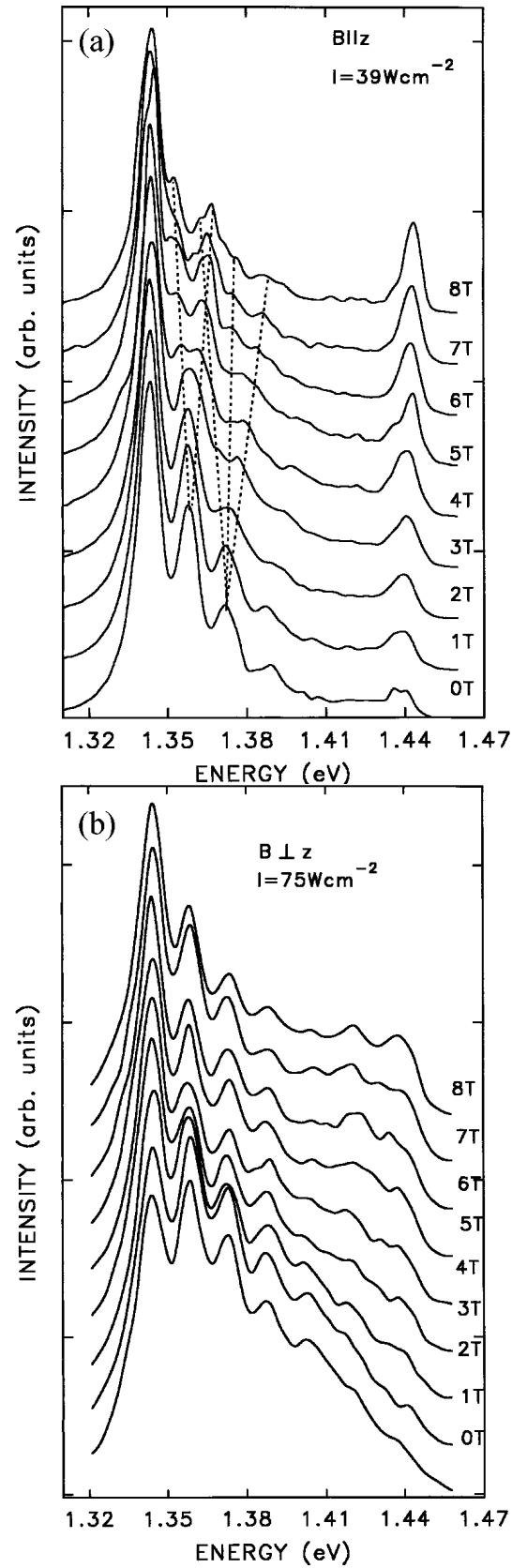

FIG. 2. Magnetoluminescence spectra in different configurations. (a) $B \|$ growth axis and $I_{\mathrm{exc}}=39 \mathrm{~W} \mathrm{~cm}^{-2}$ and (b) $B \perp$ growth axis and $I_{\text {exc }}=75 \mathrm{~W} \mathrm{~cm}^{-2}$. The dashed lines in (a) connect split transitions arising from single bands at $0 \mathrm{~T}$.

hole states the Schrödinger equation for electrons $(e)$ and holes $(h)$ in external magnetic field reads

$$
\begin{aligned}
& \left\{-\frac{\hbar^{2}}{2 m_{0}}\left[\left(\frac{\partial}{\partial z} \frac{1}{m_{z}^{(e, h)}(r, z)} \frac{\partial}{\partial z}\right)+\frac{\partial}{r \partial r}\left(\frac{r}{m_{r}^{(e, h)}(r, z)} \frac{\partial}{\partial r}\right)-\frac{1}{m_{r}^{(e, h)}} \frac{m^{2}}{r^{2}}+H_{Z}^{(e, h)}+H_{D}^{(e, h)}\right]\right. \\
& \left.+V_{\mathrm{QW}}^{(e, h)}(z)+V_{\text {strain }}^{(e, h)}(z, r)\right\} \psi_{n, m}^{(e, h)}(r, z)=E_{n, m}^{(e, h)} \psi_{n, m}^{(e, h)}(r, z),
\end{aligned}
$$


(a)

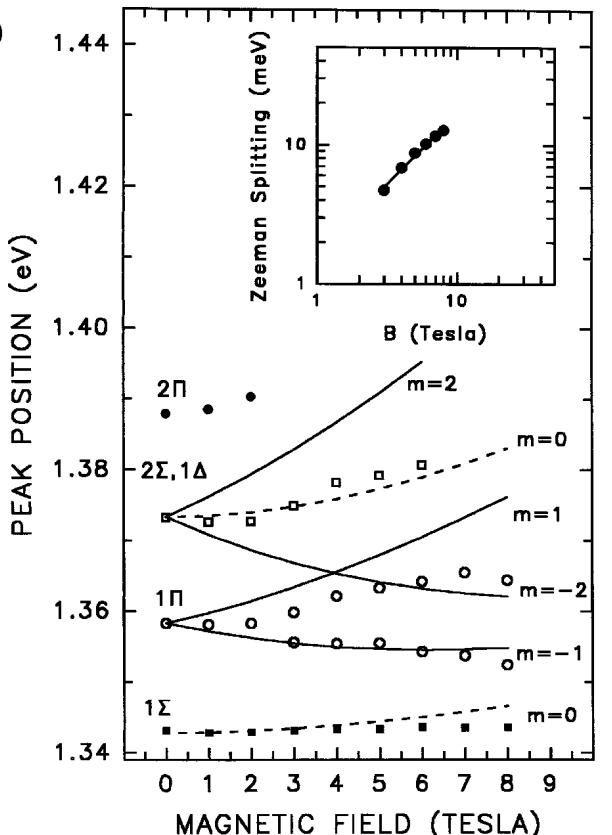

(b)

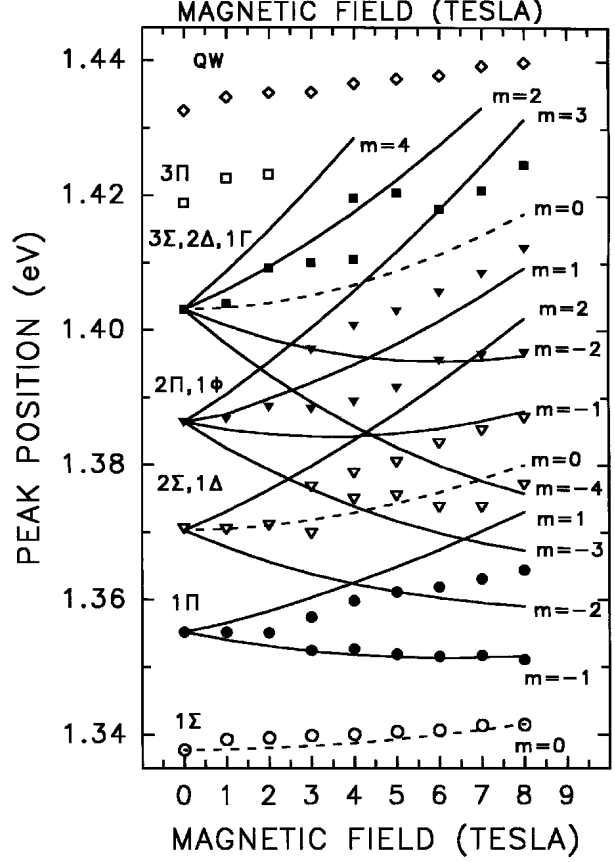

FIG. 3. (a) Fan plots extracted from the magnetoluminescence data with $B \|$ growth axis with $I_{\mathrm{exc}}=5 \mathrm{~W} \mathrm{~cm}^{-2}$ (symbols). Transitions with the same $n$ value are indicated by the same symbol. Curves are calculated according to Eqs. (2) and (3). The dashed lines indicate unsplit $(|m|=0)$ transitions. Continuous lines indicate split $(|m| \neq 0)$ transitions associated with $n \Pi^{ \pm}, n \Delta^{ \pm}, \ldots$ states. Note that symbols associated with $|m|=0$ transitions change slope at high fields due to anticrossing of different states. Inset: magnetic field dependence of the Zeeman splitting of the $1 \Pi$ transition. (b) The same as (a) but for $I_{\text {exc }}=75 \mathrm{~W} \mathrm{~cm}^{-2}$. The $\diamond ' s$ indicate the shift of the QW transition on the highest energy side of the spectrum.

where isotropic electron mass is assumed for the electron $\left(m_{r}^{e}=m_{z}^{e}\right)$. The radial and vertical heavy hole masses are given in terms of Luttinger parameters by $m_{r}^{h}=$ $1 /\left(\gamma_{1}+\gamma_{2}\right)=0.143 m_{0}$ and $m_{z}^{h}=1 /\left(\gamma_{1}-\gamma_{2}\right)$, respec- tively [14]. $\quad V_{\mathrm{QW}}^{(e, h)}(z)$ is the rectangular band edge confinement due to the quantum well, and $V_{\text {strain }}^{(e, h)}(z, r)$ is the approximately harmonic strain potential calculated by the theory of elasticity [12]. For $B \| z$ the Zeeman effect is determined by $H_{Z}^{(e, h)}=-\left(e / 2 m_{r}^{(e, h)} m_{0}\right) B L_{z}$, where $L_{z}$ is the angular momentum. The total Zeeman effect, linear in $B$, is thus given by

$$
\Delta E_{Z}=\frac{e \hbar}{2 m_{0}}\left(\frac{1}{m_{r}^{e}}+\frac{1}{m_{r}^{h}}\right) B m .
$$

The diamagnetic shift determined by the $H_{D}$ term in the Hamiltonian is given by

$$
\begin{aligned}
\Delta E_{D} & =\frac{e^{2}}{8 m_{0} c^{2}}\left(\frac{\left\langle r^{2}\right\rangle^{e}}{m_{r}^{e}}+\frac{\left\langle r^{2}\right\rangle^{h}}{m_{r}^{h}}\right) B^{2} \\
& \simeq \frac{e^{2}}{8 \mu c^{2}}\left\langle r^{2}\right\rangle B^{2} .
\end{aligned}
$$

The diamagnetic shift is quadratic in the field up to a few teslas before obtaining a linear asymptotic limit at very high field strengths [14]. If Coulomb interaction is taken into account, i.e., electrons and holes form excitons, the total diamagnetic shift of the exciton will be smaller than that forecast by Eq. (3), due to the additional confinement of the exciton wave function in the dot. This effect has been neglected in the present calculations [15].

Furthermore, the atomic part of the "atomic" Zeeman splitting should be taken into account (this is often called spin splitting, although it also involves the interaction of the orbital magnetic momentum with the magnetic field). This effect is related to the electron magnetic moment of the atomic parts of the Bloch wave functions. For electrons we have $E_{e}=g_{e}\left(e \hbar / 2 m_{0}\right) m_{s} B$, where $m_{s}= \pm 1 / 2$ and $g_{e}$ ranges between -0.598 and -0.948 [14]. For holes (at lowest order) we have $E_{h}=g_{\|}^{*}\left(e \hbar / 2 m_{0}\right) m_{j} B$, where $m_{j}= \pm 3 / 2$ and $g_{\|}^{*}$ is the atomic $g$ factor. However, even with the higher order corrections, [14], this effect is of the order of $1 \mathrm{meV}$, comparable to the quantum well case. This cannot be resolved in the luminescence spectra, and can safely be neglected here.

The results of the theoretical analysis are shown by the lines in Fig. 3, where we plot the transition energies calculated by means of Eqs. (2) and (3) for states of different $m$ value, split by the Zeeman effect. The selection rules for these transitions are $\Delta n=0$ and $\Delta m=0$ [16].

Figure 3(a) clearly shows the lifting of the degeneracy of the $1 \Pi^{ \pm}$levels. The experimentally observed splitting of this level as a function of the magnetic field is shown in the inset. The dependence is clearly linear, as expected, for the Zeeman splitting of an axially symmetric quantum dot. With increasing excitation power, it is possible to observe the splitting of several filled states (up to the fifth luminescence peak consisting of $3 \Sigma, 2 \Delta$, and $1 \Gamma$ transitions). It is worth noting that the $\Sigma$ states $(|m|=0)$ do not split and exhibit only a weak diamagnetic shift in 
the field [see dashed lines in Figs. 3(a) and 3(b)]. The fine structure of the fan plots becomes more and more complicated with increasing the quantum number $n$.

The measured diamagnetic shift of the ground level $(2 \mathrm{meV}$ at $8 \mathrm{~T}$ ) is smaller than that obtained by Eq. (3) (4 meV at $8 \mathrm{~T}$ for free carriers), due to the neglect of excitonic effects. If we add the experimental diamagnetic shift to the Zeeman term [rather than Eq. (3)], we find an even better agreement between theory and experiment. This indicates that excitonic effects could play some role in our experiment. A quantitative analysis of these effects will be presented in a forthcoming study [17]. In the opposite configuration ( $B$ perpendicular to the growth axis) [Fig. 2(b)], the average magnetic energy due to the angular part of the wave function is zero because the angle between the $z$ component of the angular momentum and the magnetic field direction is $90^{\circ}$. In this configuration the magnetic field direction is no longer a symmetry axis for the Hamiltonian, and the magnetic field induced shift of the photoluminescence bands is very small because the orbit of carriers crosses the rectangular well potential boundary along the $z$ direction. Very recently, Bayer et al. [6] have reported the magnetoluminescence spectra of QD's fabricated by shallow etching. However, their interpretation was based on a Zeeman splitting given by $(2 \hbar e / \mu) B$ (for $m= \pm 1$ transitions) which is by far larger than our experimental data and our Eq. (2).

In conclusion, we have presented the first well resolved quantitative measurement and theoretical interpretation of a fundamentally new phenomenon, the breaking of the degeneracy of quantum dots states with different angular momentum quantum numbers induced by an axial magnetic field. This Zeeman effect is peculiar to quantum confined nanostructures.

We gratefully acknowledge D. Cannoletta, A. Melcarne, and M. Corrado for expert technical help.

[1] B. Meurer et al., Phys. Rev. Lett. 68, 1371 (1992).

[2] D. Heitmann and J.K. Kotthaus, Phys. Today 46, 56 (1993); D. Heitmann, Physica (Amsterdam) 212B, 201 (1995).
[3] R. C. Ashoori et al., Phys. Rev. Lett. 68, 3088 (1992).

[4] Ch. Sikorski and U. Merkt, Phys. Rev. Lett. 62, 2164 (1989).

[5] W. Hansen et al., Phys. Rev. Lett. 62, 2168 (1989).

[6] M. Bayer et al., Phys. Rev. Lett. 74, 3439 (1995).

[7] G. W. Bryant, Phys. Rev. Lett. 59, 1140 (1987).

[8] U. Merkt et al., Phys. Rev. B 43, 7320 (1991).

[9] P. A. Maksym and T. Chakraborty, Phys. Rev. Lett. 65, 108 (1990).

[10] M. Sopanen, H. Lipsanen, and J. Ahopelto, Appl. Phys. Lett. 66, 2364 (1995).

[11] H. Lipsanen, M. Sopanen, and J. Ahopelto, Phys. Rev. B 51, 13868 (1995).

[12] J. Tulkki and A. Heinämäki, Phys. Rev. B 52, 8239 (1995).

[13] L. I. Shiff, Quantum Mechanics (McGraw-Hill, New York, 1949).

[14] Th. Wimbauer et al., Phys. Rev. B 50, 8889 (1994).

[15] Electron-electron interaction is neglected in our lowest order theoretical calculation. However, in our system, there is evidence that the experimentally observed dipoleallowed transitions can be analyzed by the one-particle picture. In fact, our present and previous (Ref. [11]) experiments show that the luminescence energies are insensitive to the state filling. Similar observations have been reported in Refs. $[2,4,9]$ by far infrared measurements in field effect quantum dots. Further, our calculations have shown that when several dot states are filled, the calculated intensity of the emission lines is very close to the measured intensity distribution under intense photoexcitation. This suggests that the transition energies of the dipole-allowed lines are insensitive to correlation. A possible explanation of this surprising result is that a properly modified generalized Kohn's theorem can be applied to our dots. Note that this theorem, which has been experimentally found to be valid for field effect quantum dots, cannot be applied as such to our dots.

[16] J. Pankove, Optical Processes in Semiconductors (Dover, New York, 1971).

[17] A coupled heavy hole-light hole band calculation has been carried out for hole states in axially symmetric quantum dot in external field. The results show that the band coupling is significant and accounting for it improves the agreement with experiment [M. Brasken, J. Tulkki, and M. Lindberg (unpublished)]. 